\title{
The Elaborate Postural Display of Courting Drosophila persimilis Flies Produces Substrate-Borne Vibratory Signals
}

\author{
Mónica Vega Hernández ${ }^{1}$. \\ Caroline Cecile Gabrielle Fabre ${ }^{1}$
}

Revised: 9 August 2016 / Accepted: 15 August 2016/

Published online: 2 September 2016

(C) The Author(s) 2016. This article is published with open access at Springerlink.com

\begin{abstract}
Sexual selection has led to the evolution of extraordinary and elaborate male courtship behaviors across taxa, including mammals and birds, as well as some species of flies. Drosophila persimilis flies perform complex courtship behaviors found in most Drosophila species, which consist of visual, air-borne, gustatory and olfactory cues. In addition, Drosophila persimilis courting males also perform an elaborate postural display that is not found in most other Drosophila species. This postural display includes an upwards contortion of their abdomen, specialized movements of the head and forelegs, raising both wings into a "wing-posture" and, most remarkably, the males proffer the female a regurgitated droplet. Here, we use high-resolution imaging, laser vibrometry and air-borne acoustic recordings to analyse this postural display to ask which signals may promote copulation. Surprisingly, we find that no air-borne signals are generated during the display. We show, however, that the abdomen tremulates to generate substrate-borne vibratory signals, which correlate with the female's immobility before she feeds onto the droplet and accepts copulation.
\end{abstract}

Keywords Biotremology $\cdot$ drosophila $\cdot$ persimilis $\cdot$ pseudoobscura $\cdot$ courtship $\cdot$ behavior . abdomen · tremulation - substrate-borne vibrations · female stationary · quivering $\cdot$ feeding . copulation

Electronic supplementary material The online version of this article (doi:10.1007/s10905-016-9579-8) contains supplementary material, which is available to authorized users.

Caroline Cecile Gabrielle Fabre

c.c.g.fabre.03@cantab.net

1 Department of Zoology, University of Cambridge, Downing Street, Cambridge CB2 3EJ, UK 


\section{Introduction}

A variety of animals perform courtship displays to attract a mate. The complexity of these displays is driven by sexual selection, which in some species has led to extraordinary patterns of male courtship (Andersson 1994). For example, during courtship, the male bird of paradise presents an iridescent chest and snaps its tail feathers rhythmically while hopping on its legs (Cooper and Forshaw 1977). In jumping spiders and wolf spiders, courting males raise their abdomen and/or legs to attract the female's attention (Waldock 1993; Hebets and Uetz 2000; Girard and Endler 2014; Girard et al. 2015). These behaviors provide visual signals to the females (Cooper and Forshaw 1977; Amundsen et al. 1997; Bennett et al. 1997; Pearn et al. 2001; Hausmann et al. 2003; Li et al. 2008; Lim et al. 2008; Uhl and Elias 2011), but, at the same time, they may also generate cues such as air-borne or substrate-borne signals (Edwards 1981; Gwynne and Dadour 1985; Maddison and Stratton 1988a, 1988b; Read and Weary 1992; Elias et al. 2003; Sivalinghem et al. 2010; Girard et al. 2011; Uhl and Elias 2011). The females use all of these pieces of information to assess males and to decide whether to accept copulation. It was long thought that the jumping spider females assessed the courting display of the males mainly visually (Foelix 1996). In some species, courting males were shown to also produce air-borne sounds [see for example (Edwards 1981; Gwynne and Dadour 1985)]. Later, the abdominal movements of the males were shown to produce substrate-borne vibrations; the transfer of these vibrations through the ground is essential for the copulation success of the males (Elias et al. 2003, 2005, 2006, 2010; Sivalinghem et al. 2010). Substrate-borne vibrational communication is widely used by animals, in particular by invertebrates, and has recently received increased attention in the literature (Virant-Doberlet and Cokl 2004; Cocroft and Rodríguez 2005; Polajnar et al. 2014; Hill and Wessel 2016; Polajnar et al. 2016; Rebar and Rodríguez 2016). Identifying and monitoring these vibrations that are imperceptible to humans requires sophisticated technologies, such as laser vibrometry (Elias et al. 2003; Cocroft and Rodríguez 2005; Girard et al. 2011).

Drosophila persimilis flies are a typical wild inhabitant of the western United States, where they breed on the infected sap of trees. Although their behaviour and ecology are not completely understood, $D$. persimilis flies are usually found together with the almost morphologically indistinguishable species Drosophila pseudoobscura (Carson 1951). Like other Drosophila species the D. persimilis males produce "standard" courtship behaviors, including the wing fluttering that produces an air-borne sound involved in species recognition (Spieth 1952; Brown 1965; Greenspan and Ferveur 2000). Remarkably, Drosophila persimilis is one of the few Drosophila species, in which males also exhibit an elaborate postural display in addition to the standard courtship steps (Spieth 1952). Some parts of this postural display were noted previously, including the upward movement of the wings ("wing-posture"), the rowing of one leg, and the dramatic rise of the abdomen and the forelegs (Brown 1965). Nevertheless, several aspects of the description and signals produced by this postural display, as well as the behavior of the female during the display, remain unknown. It has been suggested that this postural display produces mainly visual cues, and may also produce air-borne cues (Brown 1964, 1965). Here, we revisit D. persimilis courtship mating behavior and we describe the postural display using modern imaging and sophisticated recording techniques to ask which non-visual signals might promote copulation. 


\section{Materials and Methods}

\section{Flies}

Drosophila persimilis (UC San Diego Drosophila stock center, stock number 14,011-0111.00, collected from Cold Creek, California), D. pseudoobscura (UC San Diego Drosophila stock center, stock number 14,011-0121.00, collected from Tucson, Arizona) and D. melanogaster Canton-S flies were raised on standard wheatmeal medium under a $12: 12 \mathrm{~h}$ light:dark cycle and kept at $23{ }^{\circ} \mathrm{C}$ with $65 \%$ humidity. Adult flies were collected upon eclosion with light $\mathrm{CO} 2$ anesthesia. Before mating, individual males or small groups of five to ten virgin females were kept in vials with fresh food. For laser vibrometry experiments, wings were removed so as to reduce noise produced by wings during grooming and fluttering in the recordings. Filming and laser vibrometry of courting pairs were performed at a temperature of around $23{ }^{\circ} \mathrm{C}$.

\section{Recording Vibrational Signals with Laser Vibrometry}

Video and laser vibrometer recordings were conducted on a vibration-damped table in a soundproof room. Flies were placed into cylindrical chambers of approximately $10 \mathrm{~mm}$ in diameter and $9 \mathrm{~mm}$ in height. The top of this cylinder was a transparent film through which the flies were recorded using a Stingray F-33B camera (Allied Vision). One side of the cylinder consisted of a piece of thermal foil, a membrane made of silver metallised polyester material, with an albedo of approximately 0.8 (Sub Zero Technology; Leicester, UK). The beam of a OFV-534 laser vibrometer (Polytec) was directed perpendicular to the surface of this membrane. Signals were digitised with 12 bit amplitude resolution with a PCI MIO-16-E4 card (Analog Devices; Norwood, MA) and with LabView (National Instruments; Austin, TX) on a PC. Signals were transformed into .wav data with the Spike 2 (CED) or Neurolab (Hedwig and Knepper 1992) softwares. Video and laser vibrometer recordings were synchronised at the start by brief interruption of the laser path; this produces both a momentary peak in the oscillogram and a black frame in the video. Oscillograms were analysed with the Amadeus Pro (HairerSoft) and the Raven (Bioacoustics Research Program) softwares.

\section{Behavioral Recording of Courtship Assays}

Pairs of flies were tested at 7 days old when they are most active in courtship. Their behavior was recorded with a $100 \mathrm{~mm}$ macro lens and a Stingray F-033B camera (Allied Vision Technologies; Stadtroda, Germany) and acquired with the Astro IIDC (Aupperle Services and Contracting; Calgary, Canada) or the Debut Video Capture (Pro Edition) softwares into a laptop computer. High-speed videos with images captured at a rate of 1000 per second were acquired with a Photron FastcamSA3 camera (Photron (Europe) Ltd., High Wycombe, Bucks, UK). Flies were filmed in transparent plexiglass courtship chambers $(10 \mathrm{~mm}$ diameter and $9 \mathrm{~mm}$ height). Recording was started at the initiation of courtship and for approximately $600 \mathrm{~s}$, or until copulation occurred. Each pair was tested only once. Before each test, chambers were washed with ethanol and dried. 


\section{Behavior Annotations and Analysis}

Movies were annotated with the Annotation software (Peter Brodsky, version 1.3), registering all standard male courting behaviors (that include orientating toward the female, following the female, moving and vibrating the wings, extending the proboscis, licking, leg tapping, etc.) and the exhibition of the postural display (that includes the wing-posture, movements and quivers of the abdomen, movements of the head and legs, production of liquid droplets, etc.), and also whether the female was moving or stationary. The data for each movie were imported into Excel files. We generated the box plots using the R program -BoxPlotR-from the Tyers Lab (http://boxplot.tyerslab.com/). The notches designate the $95 \%$ confidence while the box limits specify the 25 th and 75th percentiles. Bold middle lines indicate medians, and crosses indicate the means. Two-sample Wilcoxon tests were used to compare female movement (moving or stationary) during the postural display and to compare the interpulse intervals of the substrate-borne vibrations generated by D. persimilis, D. pseudoobscura and D. melanogaster.

\section{Behavioral Tracking during the Postural Display}

Using Fabrice Cordelieres software, Manual Tracking, a plugin for imageJ (http:// imagej.net/Manual_Tracking) we labeled the position of the abdomen, the mouth and the front-leg over time, resulting in the formation of three super imposed lines that trace the journey of these body parts over time as the movie plays forward. In the graph, zero represents the position of the body axis before the start of the postural display and each movement is relative to that original position.

\section{Sound Recordings}

Sound was recorded using an insectavox composed of an electret microphone-amplifier board (frequency response, $50 \mathrm{~Hz}$ to $13 \mathrm{k} \mathrm{Hz}$; sensitivity, $60 \pm 3 \mathrm{~dB}$; DC $3 \mathrm{~V}-12 \mathrm{~V}$ MAX9814) attached to an iMic USB audio device (griffintechnology.com, Nashville, USA) and a laptop computer. We used the software QuickTime (Apple inc.) for sound recording. Simultaneously, videotape of courtship was performed with a portable microscope USB camera (Visual Effect, model: B003). The audio files were processed using the audio editing program Audacity (http://audacityteam.org/).

\section{Results}

\section{The male's Courtship Parade and the female's Response}

\section{The Male Display}

We recorded 40 pairs of courting $D$. persimilis flies and monitored the postural display during courtship. We refer to this postural display of courtship as PDC. Using highspeed video imaging, we broke down the PDC into 12 behaviors (Supplementary Movie S1 and associated legend) and we tracked the movements of the male's foreleg, 
abdomen and proboscis (Fig. 1, Supplementary Movie S2). The abdomen quivered while being progressively raised upward: we called this "quivering up" to differentiate it from the quivering that was observed during the standard courtship behaviors in other Drosophila species (Fig. 1; Supplementary Movies S1, S2) (Fabre et al. 2012). Sometimes, anal drops were produced (Supplementary Movie S3). The male tapped on the floor with its forelegs, and then raised one or both forelegs before moving it/them back towards the ground (Fig. 1, Supplementary Movies S1, S2, S3). While raising them up and down, the forelegs were also vibrating (Supplementary Movies S1, S2). Simultaneously, the male moved his proboscis downwards and produced a liquid droplet (Fig. 1, Supplementary Movies S1, S2, S3) presumably of regurgitated food with nutritional value (Steele 1986; Immonen et al. 2009), which was then collected by the female (Supplementary Movies S1, S3, S4).

\section{The female's Behavior during the male's Display}

During the male display, the female turned and faced the male (Supplementary Movies S1-4) but she remained mostly stationary (Fig. 2; during the PDC, the female was

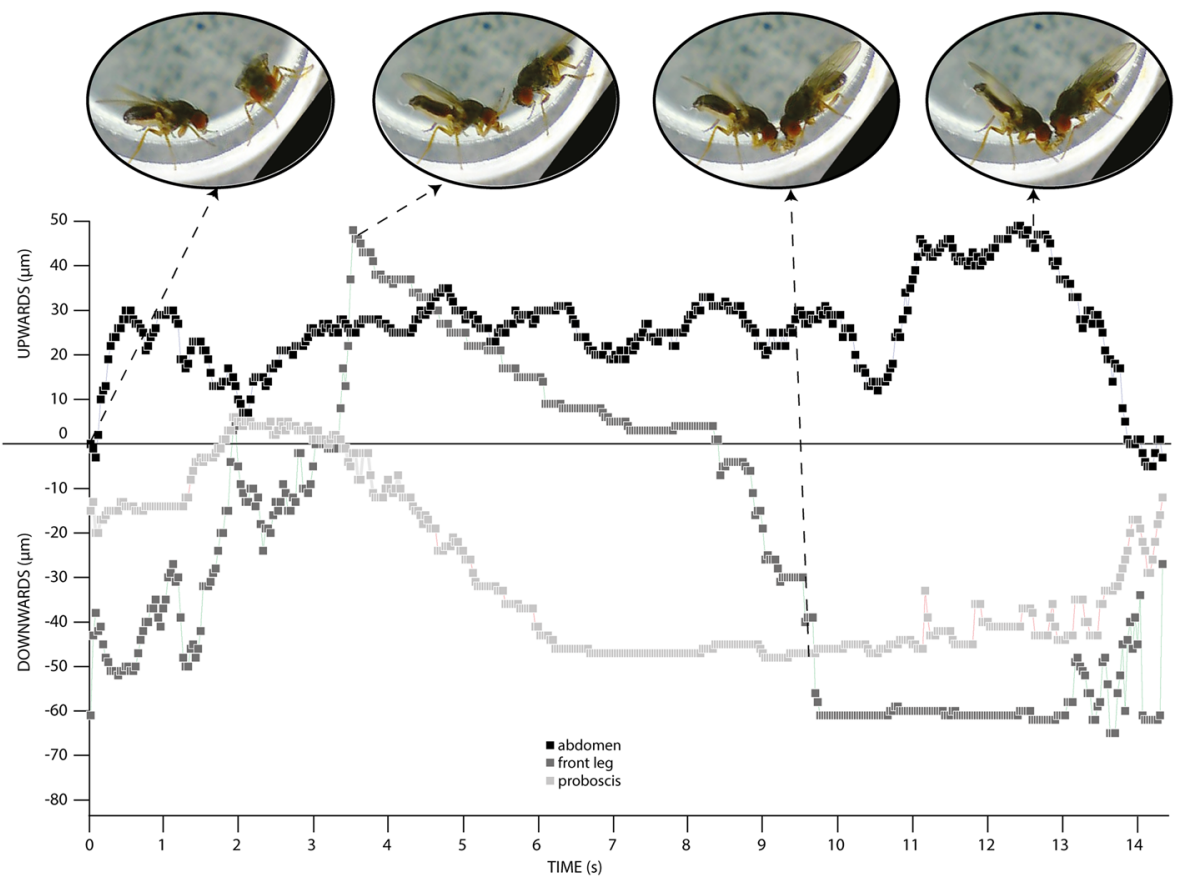

Fig. 1 Visual tracking of three main behaviors within the PDC. We tracked the position of the tip of the abdomen (in black), one of the front legs (in grey) and the tip of the proboscis (in light grey) over time for a typical display bout (Supplementary Movie S1; 30 frames per second). Selected snapshots from the video clip are shown as landmarks. We used the position of the abdomen before the display as the position zero. The $\mathrm{x}$ axis shows time in seconds. The y axis shows distance in micrometres. For abdominal movements, the trace shows quivers within the larger abdominal upward and downward movements. During these behaviors the legs are raised and then lowered to the floor. The proboscis trace shows how the male moves his proboscis towards the ground where the female can touch the drop with her front legs and feed on it 


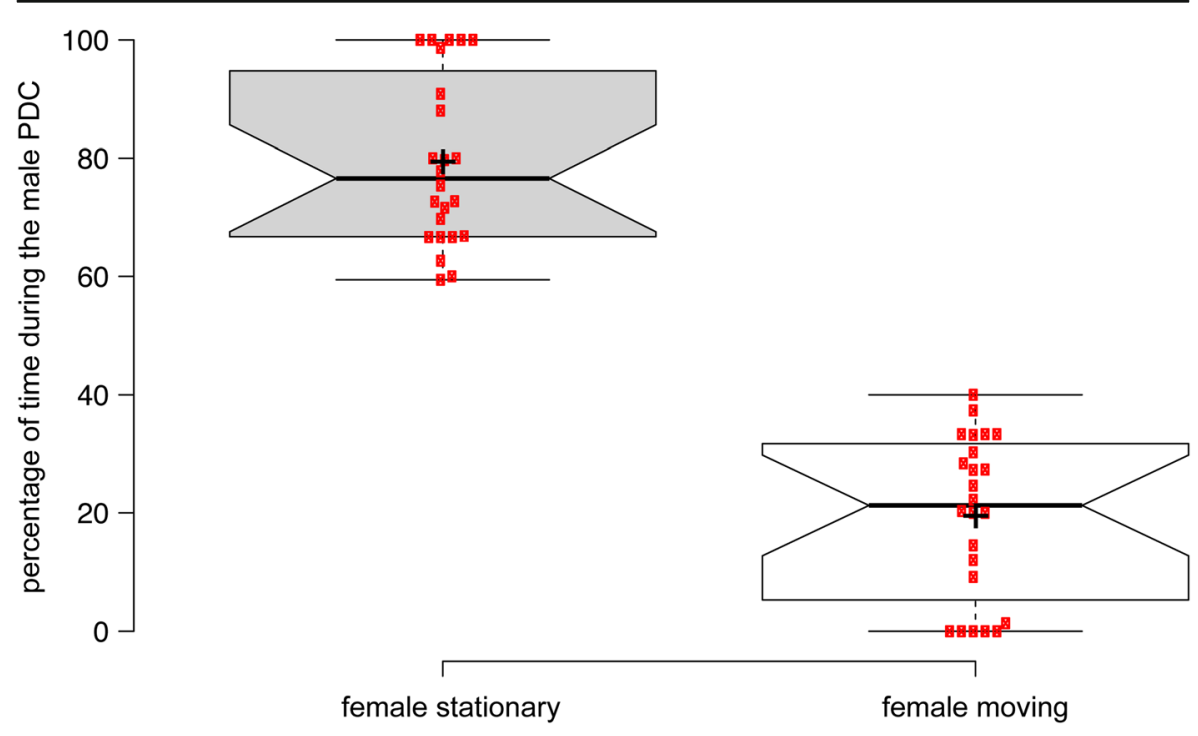

Fig. 2 Quantification of the female movements during the PDC. Ethograms for males and females were constructed from video clips of 24 courting pairs. The box plots illustrate the percentage of time where females were moving (left box plot) or stationary (right box plot) while the male was performing the PDC. Females were more likely to be stationary when males performed the PDC $(n=24 ; p=2.9 \times 10-9)$. Thick lines indicate the medians and the notches indicate $95 \%$ confidence intervals for each median; crosses indicate the mean values

stationary for about $80 \pm 3 \%$ of the time; $n=24 ; P<0.05)$. At the end of the display she approached the male to collect the droplet (Supplementary Movie S1-4). The female first touched the droplet with her forelegs (Supplementary Movies S1, S3), where flies have gustatory receptors (Montell 2009). Then, the labellar surface of her proboscis touched the labellar surface of the male's proboscis in a "kiss" and she caught the liquid droplet directly (Supplementary Movies S1, S3). Alternatively, the female sometimes picked the droplet from the floor (Supplementary Movie S4). The female did not move while eating the droplet (Supplementary Movies S1, S4) and copulation could then occur (Supplementary Movie S4). If the female moved away or appeared not to attend to him, the male interrupted his behavior and copulation did not follow (Supplementary Movie S5).

\section{Quantification of the PDC during D. persimilis Courtships}

We found that in $47 \%$ of the $D$. persimilis courting pairs $(n=40)$, the males exhibited both PDC and the standard Drosophila courtship behaviors, including fluttering one wing to produce a species-specific song, and extending the proboscis and tapping the female to assess her cuticular pheromones (see Movie S3; Spieth 1952). Within these pairs, courtship lasted an average of two minutes and the PDC occupied $\sim 13 \%$ of courtship time, predominantly occurring in the second half of courtship (not shown). Full PDC lasted between 10 and 20 s, with males exhibiting $2.6 \pm 0.7$ PDCs per courtship.

In the other $53 \%$ of courting pairs, the males did not exhibit PDC and exhibited only the standard Drosophila courtship behaviors. In these courting pairs, the male did not 
produce regurgitated food. The "textbook view" is that Drosophila courtship is stereotyped (Greenspan and Ferveur 2000) and, to our knowledge, the finding that $\sim$ half of the $D$. persimilis courting pairs behave differently to the other half has not previously been reported. We address this further in a separate report (manuscript in preparation).

\section{Substrate-Borne Vibrations Are Generated during the PDC}

Our aim was to identify which signals generated by the PDC may affect the female's behavior. It appears that the display has a visual component because the male takes up a characteristic posture and the female turns to face him (Brown 1965) (Supplementary Movies S1-3). Nevertheless, we also investigated if quivering-up of D. persimilis generated vibrations in the substrate. We used a laser vibrometer to measure the oscillations in the substrate while observing the activity of the flies with a camera. We found that D. persimilis quivering-up generated regular substrate-borne vibrations (Fig. 3, Supplementary Movie S6) with an interpulse interval of $173 \pm 4$ ms (Figs. 3 and 4).

We compared the substrate-borne vibrations of $D$. persimilis males with those of D. pseudoobscura, a co-occuring (sympatric) species. D. pseudoobscura males also performed a PDC (Brown 1964), including abdominal quivering-up movements (not shown). Laser vibrometry recording revealed that these movements generated vibrations with similar interpulse intervals to $D$. persimilis (172 $\pm 3 \mathrm{~ms} ; P=0.61$; Fig. 4). In contrast, however, these interpulse intervals were very different from those produced by D. melanogaster male quivering $(222.4 \pm 2 \mathrm{~ms}$; Fig. 4 ; $P<0.05)$ (Fabre et al. 2012; Mazzoni et al. 2013; Medina et al. 2014).

\section{Air-Borne Sounds Do not Occur during the PDC}

We examined if the wings produced air-borne cues during the wing-posture position; they move up-and-down during the display (Supplementary Movies S1-4). We did not



Fig. 3 Regular substrate-borne vibrations are generated during the abdominal quivering-up of a courting D. persimilis male. Oscillogram of substrate-borne vibrations generated during a short single bout of quivering-up (Supplementary Movie S6 (at $42 \mathrm{~s}$ )); the wings of the male were amputated. Each pulse is about $5 \mathrm{~ms}$ long and of amplitude below $400 \mu \mathrm{m} / \mathrm{s}$. Walking and grooming generate the larger amplitude signals visible before and after the quivering bout 


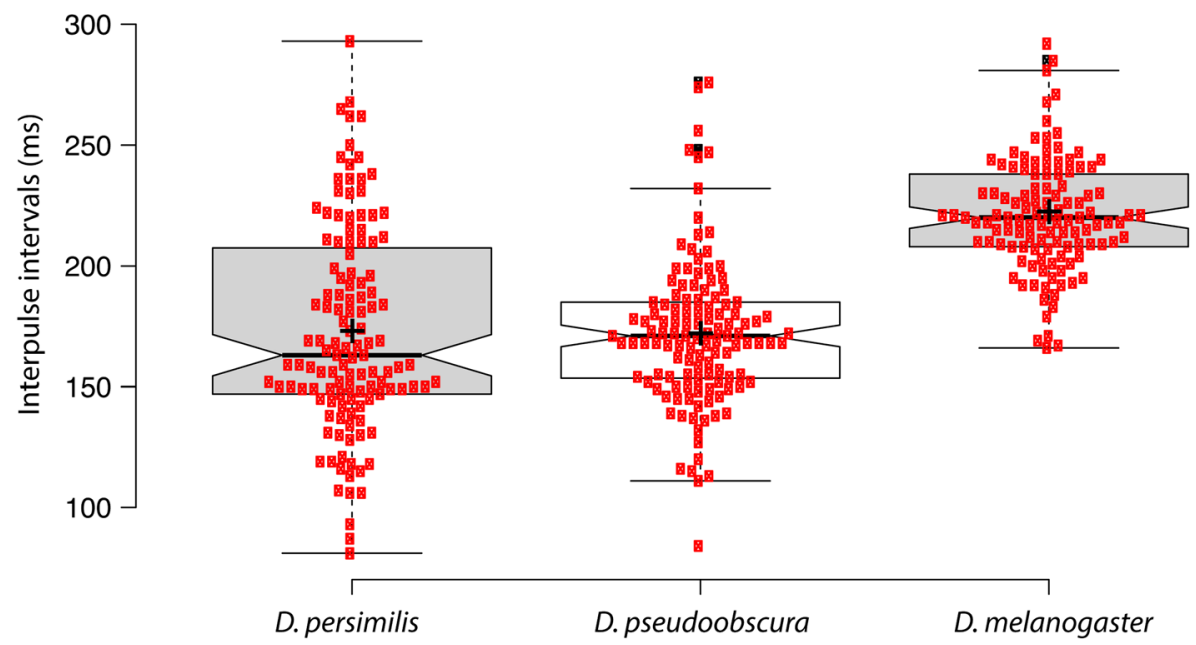

Fig. 4 Interpulse intervals of the substrate-borne vibrations generated by quivering-up of $D$. persimilis and D. pseudoobscura males during the PDC and by quivering of D. melanogaster males during the standard courtship. Boxplots of the interpulse intervals (in $\mathrm{ms}$ ) for the substrate-borne vibrations of wild-type $D$. persimilis males paired with wild-type $D$. persimilis virgin females $(n=124$ pulses recorded, column 1$)$; of wild type $D$. pseudoobscura males paired with wild type $D$. pseudoobscura virgin females $(n=125$ pulses recorded; column 2); and wild type Canton S D. melanogaster males paired with Canton $\mathrm{S}$ virgin females ( $n=117$ pulses recorded; column 3). Data are shown for 3 individuals for each different species. There were no significant differences in the mean interpulse interval for $D$. persimilis and $D$. pseudoobscura species $(P=0.61)$. However, both showed significant differences with $D$. melanogaster mean interpulse interval $(P=2.2 \times 10-16)$. Red dots show all data points. Notches indicate $95 \%$ confidence intervals of each median, thick lines indicate the medians, and crosses indicate the mean values

detect any air-borne sound generated during the PDC, suggesting that these wing movements are silent (Fig. 5, Supplementary Movie S7). We did record, however, the pulse song generated by the male wing fluttering during the standard courtship behaviors (Waldron 1964; Noor and Aquadro 1998) (Fig. 5, Supplementary Movie S7).



Fig. 5 Insectavox sound recordings during $D$. persimilis courtship. Air-borne sounds were recorded during courtship using an insectavox (see materials and methods). Bouts of wing fluttering (WF) of $D$. persimilis males produced pulse song (solid black lines; supplementary Movie S7). Inset (yellow box) shows pulse song in more detail. The pulses have a frequency of around $200 \mathrm{~Hz}$. After three bouts of WF, the male performed the PDC (indicated by a blue rectangle and a dotted line underneath). No characteristic air-borne signals were produced during the PDC (Supplementary Movie S7) 


\section{Discussion}

Our results show that the postural display of courtship (PDC) produced by D. persimilis males is highly complex and includes twelve behaviors, some of which are performed simultaneously. In addition, the males use substrate-borne vibratory signals during the PDC, which co-occur with the upward and downward movement of the quivering abdomen. During these vibrations, the female remains stationary. The female is then proffered with a droplet that might be nutritive (Steele 1986; Immonen et al. 2009), which she feeds on while remaining immobile. Altogether, these results suggest that the male's PDC endeavours to secure stationary females, as this may promote easier copulation (Ferveur 2010; Fabre et al. 2012). We do not know what is contained in the liquid droplet, although it is possible that it may promote egg laying (Steele 1986; Immonen et al. 2009). Females often touch this droplet with their front legs and this might allow them to assess its nutritive value or to evaluate male fitness or some other characteristic (Ferveur 2010; Tabadkani et al. 2012; Clutton-Brock and Huchard 2013). We were not able to find substrate-borne vibratory signals associated with the movement and vibration of the male's forelegs. It is possible that the signals generated by the leg vibrations are concealed by the signals produced by the abdominal vibrations, as they are synchronous. Alternatively, the leg vibrations might only provide visual signals to the females. Similarly, during the parade of courting male jumping spider, Habronattus dossenus, substrate-borne vibratory signals were recorded during the abdominal vibratory movements, but not during some of the leg gestures (Elias et al. 2003).

The substrate-borne signals produced by $D$. persimilis males are different to those of D. melanogaster, indicating that the substrate-borne vibrations may be involved in species discrimination. However, the substrate-borne signals produced by $D$. persimilis males are similar to those of $D$. pseudoobscura. D. persimilis and D. pseudoobscura species do not produce hybrids in the wild (Dobzhansky and Epling 1944), even though they are morphologically similar, have similar cuticular hydrocarbons (Noor 1996), and produce similar substrate-borne signals (this study). Species boundaries are likely maintained by differences in the length of their genitalia (Rizki 1950) and the airborne courtship songs that they produce (Brown 1965; Noor and Aquadro 1998); their evolutionary divergence has been much studied [see for example (Merrell 1954; Noor and Aquadro 1998; Williams et al. 2001; Noor et al. 2007)]. Our results suggest that the substrate-borne vibrations generated by $D$. persimilis and D. pseudoobscura males are unlikely to be involved in the discrimination between these two species and might instead constitute a common pacifying effect on the females in both species (Fabre et al. 2012).

It is likely that the female is attracted to the visual gestures of the PDC as she turns to face and observe the displaying male. Courtship in the dark would help reveal whether the substrate-borne vibratory signals are sufficient to account for the female's attention during the PDC. Unfortunately, we obtained very low levels of $D$. persimilis and D. pseudoobscura courtship in the dark (not shown), something that was previously noted (Spieth 1952). This suggests that vision is important for their courtship, but it also makes it difficult to discriminate between the contribution of visual and substrate-borne signals for the female's receptivity. Of note, however, is that the substrate-borne signals 
in some species of jumping spiders are more important than the visual displays for copulation success (Elias et al. 2005, 2010).

The abdominal quivering-up exhibited in D. persimilis during the PDC was reminiscent of the abdominal quivering reported in D. melanogaster and other Drosophila species during standard courtship behaviours (Fabre et al. 2012; Mazzoni et al. 2013) and these abdominal quivers all generate vibrations in the substrate. Unlike other species, however, D. persimilis males quiver while moving the abdomen upwards, away from the ground (Fabre et al. 2012; Mazzoni et al. 2013). When they were first identified it was unclear whether the substrate-borne vibrations generated by $D$. melanogaster males might depend on direct striking of the tip of the abdomen on the substrate (i.e. percussions) or whether they might be transmitted to the ground through the legs (i.e. tremulations) (Busnel et al. 1955; Morris 1980; Lasbleiz et al. 2006; Hill 2008; Fabre et al. 2012; Mazzoni et al. 2013). Clearly in $D$. persimilis abdominal striking on the substrate is not required to produce the substrate-borne vibrations. Close inspection of D. melanogaster abdominal quivers also suggests that the abdomen does not touch the substrate (Fabre et al. 2012; Mazzoni et al. 2013). Most likely therefore all abdominal quivers in Drosophila species are tremulations that are relayed to the substrate through the legs of the male or by other unknown mechanisms (Virant-Doberlet and Cokl 2004; Fabre et al. 2012; Mazzoni et al. 2013). The similar movements of tremulation (Busnel et al. 1955) through oscillation of body parts found in other animals for substrate-borne vibration production are thought to occur by coupling to the substratum through the adhesive hairs on the tips of the legs (Rovner 1980; Uetz and Stratton 1982; Aicher et al. 1983; Aicher and Tautz 1990; Dierkes and Barth 1995; Barth 2002; Elias et al. 2003; Uhl and Elias 2011).

Such a complex postural display that associates visual gestures and substrateborne vibratory signals is unprecedented in Drosophila. It is reminiscent of the complex parades displayed by larger arthropods such as the jumping spiders (Elias et al. 2003; Hill 2008). It also adds to the growing list of examples showing that vibratory signalling is a mode of communication that is widespread among animals, in particular among arthropods, so much so that it has given rise to an entire field of study now known as "biotremology" (Endler 2014; Hill and Wessel 2016). It is unclear what triggers the PDC in D. persimilis males and why we observed it only in half of the courting pairs. Such an effortful display must presumably provide advantages to the male, but it is unknown how much more successful males producing PDCs are versus those that do not display. Further studies looking into different status of the courting pairs and different courting contexts should elucidate the favourable conditions in which $D$. persimilis males choose to exhibit the PDC during courtship, in addition to the standard courtship behaviors. Such studies will determine what benefits, if any, they gain from producing the PDC.

Acknowledgments We thank P. A. Lawrence and B. Hedwig for their support and our reviewers for helpful comments; the UC San Diego Drosophila Stock Center for flies; M. Burrows, S. Laughlin, J. Casal, T. Bailey, C. Spottiswoode and P. Tate for help with experimental set-ups, softwares and discussions. C.C.G.F. was supported by a Hanne and Torkel Weisfogh research award and an Isaac Newton Trust fellowship. This work was supported by the Wellcome Trust Investigator Awards to P. A. Lawrence WT096645MA and WT107060AIA. 
Open Access This article is distributed under the terms of the Creative Commons Attribution 4.0 International License (http://creativecommons.org/licenses/by/4.0/), which permits unrestricted use, distribution, and reproduction in any medium, provided you give appropriate credit to the original author(s) and the source, provide a link to the Creative Commons license, and indicate if changes were made.

\section{References}

Aicher B, Tautz J (1990) Vibrational communication in the fiddler crab, Uca pugilator. 1. Signal transmission through the substratum. J Comp Physiol A 166:345-353

Aicher B, Markl H, Masters WM, Kirschenlohr HL (1983) Vibration transmission through the walking legs of the fiddler crab, Uca pugilator (Brachyura, Ocypodidae) as measured by laser Doppler vibrometry. $J$ Comp Physiol 150:483-491

Amundsen T, Forsgren E, Hansen LT (1997) On the function of female ornaments: male bluethroats prefer colourful females. Proc R Soc Lond B Biol Sci 264(1388):1579-1586

Andersson M (1994) Sexual selection Monographs in Behavior and Ecology. Princeton University Press, Princeton

Barth FG (2002) A Spider's World: Senses and Behavior. Springer-Verlag, Berlin

Bennett AT, Cuthill IC, Partridge JC, Lunau K (1997) Ultraviolet plumage colors predict mate preferences in starlings. Proc Natl Acad Sci U S A 94(16):8618-8621

Brown RG (1964) Courtship behaviour in the Drosophila obscura group. I: pseudoobscura. Behaviour 23(1/2):61-106

Brown RG (1965) Courtship behaviour in the Drosophila obscura group. II. Comparative Studies. Behaviour 25(3):281-323

Busnel R, Pasquinelly F, Dumortier B (1955) La tremulation du corps et la transmission aux supports des vibrations en résultant comme moyen d'information à courte portée des Ephippigères mâles et femelles. Bulletin de la Societé Zoologique de France 80:18-22

Carson HL (1951) Breeding sites of Drosophila pseudoobscura and Drosophila persimilis in the transition zone of the Sierra Nevada. Evolution 5(2):91-96

Clutton-Brock TH, Huchard E (2013) Social competition and selection in males and females. Philos Trans $R$ Soc Lond Ser B Biol Sci 368(1631):20130074

Cocroft RB, Rodríguez RL (2005) The Behavioral Ecology of Insect Vibrational Communication. Bioscience 55(4):323-334

Cooper WT, Forshaw JM (1977) The Birds of Paradise and Bower Birds. Collins, Boston

Dierkes S, Barth FG (1995) Mechanism of signal production in the vibratory communication of the wandering spider Cupiennius getazi (Arachnida, Araneae). J Comp Physiol A 176:31-44

Dobzhansky T, Epling C (1944) Contributions to the genetics, taxonomy and ecology of Drosophila pseudoobscura and its relatives, Carnegie Inst., Washington, DC. Publ 554:47-144

Edwards G (1981) Sound production by courting males of Phidippus mystaceus (Araneae: Salticidae). Psyche 88(3-4):199-214

Elias DO, Mason AC, Maddison WP, Hoy RR (2003) Seismic signals in a courting male jumping spider (Araneae: Salticidae). J Exp Biol 206(22):4029-4039

Elias DO, Hebets EA, Hoy RR, Mason AC (2005) Seismic signals are crucial for male mating success in a visual specialist jumping spider (Araneae: Salticidae). Anim Behav 69(4):931-938

Elias DO, Hebets EA, Hoy RR (2006) Female preference for complex/novel signals in a spider, Behav. Ecol 17(5):765-771

Elias DO, Sivalinghem S, Mason AC, Andrade MC, Kasumovic MM (2010) Vibratory Communication in the Jumping Spider Phidippus clarus: Substrate-borne Courtship Signals are Important for Male Mating Success. Ethology 116(10):990-998

Endler JA (2014) Foreward: The emerging field of tremology. Springer, Heidelberg

Fabre CC, Hedwig B, Conduit G, Lawrence PA, Goodwin SF, Casal J (2012) Substrate-borne vibratory communication during courtship in Drosophila melanogaster. Curr Biol 22(22):2180-2185

Ferveur JF (2010) Drosophila female courtship and mating behaviors: sensory signals, genes, neural structures and evolution. Curr Opin Neurobiol 20(6):764-769

Foelix R (1996) Biology of spiders. Oxford University Press, New York

Girard MB, Endler JA (2014) Peacock spiders. Curr Biol 24(13):R588-R590 
Girard MB, Kasumovic MM, Elias DO (2011) Multi-modal courtship in the peacock spider, Maratus volans (OP-Cambridge, 1874. PLoS One 6(9):e25390

Girard MB, Elias DO, Kasumovic MM (2015) Female preference for multi-modal courtship: multiple signals are important for male mating success in peacock spiders. Proc Biol Sci 282(1820):20152222

Greenspan RJ, Ferveur JF (2000) Courtship in drosophila. Annu Rev Genet 34:205-232

Gwynne D, Dadour I (1985) A new mechanism of sound production by courting male jumping spiders (Araneae: Salticidae, Saitis Michaelseni Simon. J Zool 207(1):35-42

Hausmann F, Arnold KE, Marshall NJ, Owens IP (2003) Ultraviolet signals in birds are special. Proc Biol Sci 270(1510):61-67

Hebets EA, Uetz GW (2000) Leg ornamentation and the efficacy of courtship display in four species of wolf spider (Araneae: Lycosidae. Behav Ecol Sociobiol 47:280-286

Hedwig B, Knepper M (1992) NEUROLAB, a comprehensive program for the analysis of neurophysiological and behavioural data. $J$ Neurosci Methods 45(1-2):135-146

Hill PSM (2008) Vibrational communication in animals. Harvard University Press, Cambridge

Hill PSM, Wessel A (2016) Biotremology. Curr Biol 26(5):R187-R191

Immonen E, Hoikkala A, Kazem AJN, Ritchie MG (2009) When are vomiting males attractive? Sexual selection on condition-dependent nuptial feeding in Drosophila subobscura. Behav Ecol 20(2):289-295

Lasbleiz C, Ferveur JF, Everaerts C (2006) Courtship behaviour of Drosophila melanogaster revisited. Anim Behav 72:1001-1012

Li J, Zhang Z, Liu F, Liu Q, Gan W, Chen J, Lim ML, Li D (2008) UVB-based mate-choice cues used by females of the jumping spider Phintella vittata. Curr Biol 18(9):699-703

Lim M, Li J, Li D (2008) Effect of UV-reflecting markings on female mate-choice decisions in Cosmophasis umbratica, a jumping spider from Singapore. Behav Ecol 19:61-66

Maddison WP, Stratton GE (1988a) A common method of sound production by courting jumping spiders (Araneae, Salticidae. J Arachnol 16(2):267-269

Maddison WP, Stratton GE (1988b) Sound production and associated morphology in male jumping spiders of the Habronattus agilis species group (Araneae, Salticidae. J Arachnol:199-211

Mazzoni V, Anfora G, Virant-Doberlet M (2013) Substrate vibrations during courtship in three drosophila species. PLoS One 8(11):e80708

Medina I, Casal J, Fabre CC (2014) Do circadian genes and ambient temperature affect substrate-borne signalling during Drosophila courtship? Biol Open 4(11):1549-1557

Merrell DJ (1954) Sexual isolation between Drosophila persimilis and Drosophila pseudoobscura, Amer. Nat 88(839):93-99

Montell C (2009) A taste of the drosophila gustatory receptors. Curr Opin Neurobiol 19(4):345-353

Morris GK (1980) Calling display and mating-behavior of Copiphora Rhinoceros Pictet (Orthoptera, Tettigoniidae. Anim Behav 28:42-51

Noor MAF (1996) Absence of species discrimination in Drosophila pseudoobscura and D. persimilis males. Anim Behav 52:1205-1210

Noor MAF, Aquadro CF (1998) Courtship songs of Drosophila pseudoobscura and D. persimilis: analysis of variation. Anim Behav 56(1):115-125

Noor MA, Garfield DA, Schaeffer SW, Machado CA (2007) Divergences between the Drosophila pseudoobscura and $D$. persimilis genome sequences in relation to chromosomal inversions. Genetics 177(3):1417-1428

Pearn SM, Bennett AT, Cuthill IC (2001) Ultraviolet vision, fluorescence and mate choice in a parrot, the budgerigar Melopsittacus undulatus. Proc R Soc Lond Ser B Biol Sci 268(1482):2273-2279

Polajnar J, Eriksson A, Rossi Stacconi MV, Lucchi A, Anfora G, Virant-Doberlet M, Mazzoni V (2014) The process of pair formation mediated by substrate-borne vibrations in a small insect. Behav Process 107:68-78

Polajnar J, Eriksson A, Virant-Doberlet M, Mazzoni V (2016) Mating disruption of a grapevine pest using mechanical vibrations: from laboratory to the field. J Pest Sci:1-13

Read AF, Weary DM (1992) The evolution of bird song: comparative analysis. Philos Trans R Soc B 338

Rebar D, Rodríguez RL (2016) Males adjust their signalling behaviour according to experience of male signals and male-female signal duets. J Evol Biol 29(4):766-776

Rizki MTM (1950) Morphological differences between two sibling species, Drosophila pseudoobscura and Drosophila persimilis. Proc Natl Acad Sci U S A 37:156-159

Rovner JS (1980) Vibration in Heteropoda venatoria (Sparassidae) - a 3rd method of sound production in spiders. J Arachnol 8:193-200

Sivalinghem, S., Kasumovic, M. M., Mason, A. C., Andrade, M. C. and Elias, D. O. (2010) Vibratory communication in the jumping spider Phidippus clarus: polyandry, male courtship signals, and mating success, Behav. Ecol: arq150. 
Spieth HT (1952) Mating behavior within the genus Drosophila (Diptera. Bull Am Mus Nat Hist 99(7)

Steele RH (1986) Courtship feeding in Drosophila subobscura. I. The nutritional significance of courtship feeding. Anim Behav 34:1087-1098

Tabadkani SM, Nozari J, Lihoreau M (2012) Inbreeding and the evolution of sociality in arthropods. Naturwissenschaften 99(10):779-788

Uetz GW, Stratton GE (1982) Acoustic communication and reproductive isolation in spiders. Princeton University Press, Princeton

Uhl G, Elias D (2011) Spider behaviour: flexibility and versability. Cambridge University Press, Cambridge Virant-Doberlet M, Cokl C (2004) Vibrational communication in insects. Neotrop Entomol 33:121-134

Waldock J (1993) Peacocks of the spider world, Aust. Nat Hist 24(5):10-11

Waldron, I. (1964) Courtship sound production in two sympatric sibling drosophila species, Science 144(3615): 191-3.

Williams MA, Blouin AG, Noor MA (2001) Courtship songs of Drosophila pseudoobscura and D. persimilis. II. Genetics of species differences. Heredity $86: 68-77$ 